\title{
EPIDEMIC INTERFERENCE IN THE ERROR PROBABILITY OF A 5G CELLULAR SYSTEM
}

\author{
Tulio Freitas Simões de Castro ${ }^{1}$; Esmael Caliman Filho ${ }^{1}$; Osmar Pinto Oliveira Junior ${ }^{1}$; Rafael \\ Andrade Vieira ${ }^{1}$; Marcelo Sampaio de Alencar ${ }^{1}$; Ana Tereza Andrade Borba ${ }^{1}$; Luiz Fernando \\ Taboada Gomes Amaral ${ }^{1}$
}

${ }^{1}$ Embedded Electronics Area; SENAI CIMATEC, Brazil.

\begin{abstract}
This article introduces concepts of 5G networks and presents a mathematical model of network interference caused by a sudden increase in the number of users. Fluctuations of the number of users cause a non stationary network traffic and the usual tools have little use. So, the proposed model is obtained through the stochastic integration of the differential equation that describes the treated non stationary phenomena. Monte Carlo simulations were made, then, to be compared with the analytical results, showing that the simulations corresponded to the expected. The stochastic integral revealed to be efficient to attack the problem. Therefore, with the proper modelling, it is possible to research techniques for mitigating the epidemic interference.
\end{abstract}

Keywords: Wireless communications, 5G networks; stochastic integration; interference analysis; massive loT.

\section{EFEITO DA INTERFERÊNCIA EPIDÊMICA NA PROBABILIDADE DE ERRO DE UM SISTEMA CELULAR 5G}

Resumo: Este artigo introduz conceitos de redes $5 \mathrm{G}$ e apresenta um modelo matemático de interferência epidêmica na rede causada pelo crescimento abrupto do número de usuários. Flutuações no número de usuários causam um tráfego não estacionário na rede e as ferramentas usuais têm pouca utilidade. Portanto, o modelo proposto é obtido a partir da integração estocástica da equação diferencial que descreve o fenômeno tratado, não estacionário. Foram feitas então simulações de Monte Carlo para comparação com os resultados analíticos, mostrando que as simulações corresponderam com o esperado. A integral estocástica se mostrou eficiente para atacar o problema. Assim, com a modelagem apropriada, é possível, em trabalhos futuros, pesquisar técnicas para mitigar a interferência epidêmica.

Palavras-chave: Comunicações sem fio; redes 5G; integração estocástica; análise de interferência; loT massivo. 


\section{INTRODUCTION}

The increase of data traffic in cellular networks motivates the design of more efficient networks with higher capacity [1]. The improvement on the mobile networks advances the urban environment and also opens space for industrial applications, which includes Internet of Things (IoT).

A 5G network is divided into two main parts: the 5G Radio Access Network (RAN), which is responsible for the wireless network communication interface aspects, and the 5G Core Network (CN), which is the computational center of the network, responsible for security aspects, mobility and external medium access, such as, the Internet.

\subsection{Description of $5 G$ technology}

The 5G technology implements many functionalities to expand the use of the network, allowing new applications, which were not viable due to limitations of the previous mobile networks. The Third Generation Partnership Project (3GPP), a standardizing organization for telecommunications, periodically releases documents with detailed directions about new technology implementation. In 3GPP release 15, the 5G New Radio (NR) is specified in two architectures: non-standalone (NSA) and standalone $(\mathrm{SA})$.

Compatibility with previous technologies is not a requisite of the $5 \mathrm{G}$ network, however, NSA architecture relies on harmonic coexistence between $5 \mathrm{G}$ and LongTerm Evolution (LTE). The NSA architecture can be seen in Fig. 1 (a), in which the 5G radio access network (5G RAN), represented by the radio base station nodes gNB (next Generation Node B), requires an existing LTE radio base station eNB (evolved Node B) in order to transmit the control signals and access LTE core network.

This architecture is interpreted as an intermediary form of development to reach the future Stand Alone (SA) architecture, a uniquely 5G network. In SA architecture, as shown in Fig. 1 (b), it is possible to observe that one single base station transmits control and data signals, making it possible to reach the maximum capacity of a $5 \mathrm{G}$ network. One important fact is that $5 \mathrm{G}$ Core also permits connection to LTE radio base stations, which facilitates compatibility with the previous generations of technology.

Figure 1 - 5G New Radio Architectures

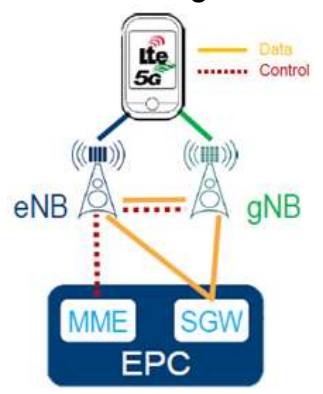

(a) 5 G NR (NSA)

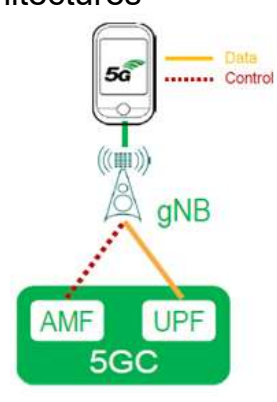

(b) 5 G NR SA

Source: Frederico \& Sabino (2019) [2] 
The 5G technology aims to satisfy some Key Performance Indexes (KPI), to satisfy more intelligent, demanding, reliable, safe and sustainable applications by new enhancements.

Many techniques were implemented in $5 \mathrm{G}$ in order to increase the signal transmission efficiency and number of simultaneously connected devices, such as Quadrature Amplitude Modulation (QAM), Orthogonal Frequency Division Multiple Access (OFDMA), massive Multiple Input Multiple Output (m-MIMO) techniques along with beamforming. Network slicing was also implemented, which is a technique to separate virtual networks within the same physical network.

The modulation techniques for 5G NR are, mainly, 16QAM, 64QAM, 256QAM and Quadrature Phase Shifting Keying (QPSK), and this last modulation scheme is used for broadcast and downlink or uplink control. These techniques make it possible to associate many bits to a single symbol.

Besides the implemented techniques, the core of $5 \mathrm{G}$ has changed the architecture to a Service Based Architecture (SBA), which makes it easier to virtualize the network functions. The most important entities are: Access and Mobility Management Function (AMF), which manages registers, connections and mobility; User Plane Function (UPF), which performs the routing and packet forwarding, interconnection with the data network and application of policies; and the Session Management Function (SMF), in charge of user data traffic related signaling. By utilizing new network management tools, the fifth generation brings along with it more data traffic reliability when compared to the previous cellular technologies.

\section{METHODOLOGY}

It is usual to assume stationarity in the analysis of symbol or bit error probability, because a non-stationary environment would complicate matters in terms of mathematical modelling.

But, in cellular communication systems, the interference is usually dependent on the state of the network. For example, if the users are accessing the network early morning, or leaving the telephone system late night, the traffic is clearly non-stationary, and so is the interference [3].

For non-stationary processes, such as in the case of a sudden increase in telephone traffic, the usual linear tools, such as basic stochastic processes, are less useful, but stochastic integration can be used to attack the problem, based on a stochastic differential equation formulation, in place of the usual mathematical approach that uses correlation.

\subsection{Computation of the Interference Instantaneous Power}

The stochastic processes related to a cellular communications system that enters an epidemic state, when most users rush to place calls, are usually nonstationary, which means that their statistical averages, or moments, also vary with time.

This makes it difficult to use the traditional correlation analysis to treat them. In this case, stochastic integration is a useful tool to attack the problem, to obtain a problem formulation, from the modelling of the interference based on a stochastic differential equation. 
The stochastic calculus began with the study and modelling of market prices, this is, the fluctuation of the stock value as a function of time. In this case, the investors work based on the variation of the potential gain or loss, $d X(t)$, as a proportion of the invested sum $X(t)$.

In the present case, in fact, what matters is the relative instantaneous power, $d P(t) / P(t)$, of a certain signal, as it reacts to the channel fluctuations. This means that the power variation should be proportional to a Wiener process $\$ W(t) \$$, which is a function of the interference in the channel, that is, it increases with the rise in interference, [4]

$$
d P(t)=\beta P(t) d W(t)
$$

which is an informal manner to express the corresponding integral equation,

$$
P(t+\tau)-P(t)=\beta \int_{t}^{t+\tau} P(u) d W(u)
$$

An immediate question associated to the equation solution is related to the nondifferentiability of a Wiener process $W(t)$, at any point in time. A way to circle the problem has been found, and is known as the theory of stochastic integrals, or the study of stochastic differential equations $[5,6]$.

The Itô general stochastic equation is given by

$$
d P(t)=a[P(t), t] d t+b[P(t), t] d W(t),
$$

in which $\alpha P(t) d t$ is the drift function, or model trend, and $\beta P(t) d W(t)$ is the dispersion function, or volatility, of the stochastic process.

\subsection{Model for the Interference Instantaneous Power}

When formulating a stochastic model to represent the interference variation along with time, it is important to consider that the interference increase rate is proportional to the existing amount of interference, because, as in an epidemic, the interference grows at a rate that is proportional to the number of users [7].

On the other hand, the incremental variation of the interference is proportional to the differential variation $d W(t)$ of a stochastic process, $W(t)$, which usually has a Gaussian distribution, multiplied by the total power of the active users, $\$ \mathrm{P}(\mathrm{t}) \$$, and adjusted by the parameter $\beta$, which remains to be found, based on the channel specifications.

The process $W(t)$ is the result of a combination of all interference factors, $W_{i}(t)$, which can be found in the cellular communication system. Thus, by the Central Limit Theorem, $W(t)$ has a Gaussian probability distribution.

Combining both assumptions, results in the following stochastic differential equation 


$$
d P(t)=\alpha P(t) d t+\beta P(t) d W(t),
$$

In order to solve the stochastic differential equation obtained with the interference model, using the generic formulation for stochastic differential equations, one must consider that the drift function is proportional to the stochastic process, this is, $a[P(t), t]=\alpha P(t)$, that the dispersion function is modelled as $b[P(t), t]=\beta P(t)$, and that one can use the Itô formula for the logarithm function $f(x, t)=\log (x)$.

Following the procedure established by Itô, for the solution of the equation, one obtains

$$
P_{E}=P(0) e^{\left(\alpha-\frac{\beta^{2}}{2}\right) t+\beta W(t)} .
$$

This solution shows, for the proposed stochastic process, that the interference power grows exponentially in time, controlled by the parameters $\alpha$ and $\beta$, that remain to be determined. It is possible to note a random variation on the curve, as a result of the stochastic process $W(t)$.

For a stochastic process, $W(t)$, that corresponds to the total interference, if its distribution is Gaussian, it is possible to compute the associated interference distribution, using the transformation of probability density function mathematical operation.

Note that the stochastic process is non-stationary, in the long run, because its average value depends on time. Besides, the probability distribution of process $P(t)$, that represents the interference, is not Gaussian, as is usually assumed in the computation of the error probability, using the usual theory of stochastic processes.

\subsection{The influence of the combined interference}

As discussed in the previous section, the stochastic process $W(t)$ results from the combination of several interferers, $W_{i}(t)$, as the users decide to place calls at the same time, or in the same time interval.

The probability distribution of the interfering power, $P(t)$, can then be found, giving

$$
f_{P}(p)=\frac{P_{0} e^{-\frac{\left(\ln p-\ln P_{0}\right)^{2}}{2 \beta^{2} \sigma_{W}^{2}}}}{\beta p w_{w} \sqrt{2 \pi}} \text {, se } p>0 \text {, e nulo no caso } p \leq 0,
$$

\section{RESULTS AND DISCUSSION}

The random process that represents the interference in a communication system that suffers from a pandemic attack, has a Lognormal probability distribution, instead of the usual assumption of a Gaussian distribution.

Fig. 2 (b) illustrates the effect of the interference caused by the Lognormal probability density function (pdf), on a BPSK signal, compared to the effect of Gaussian interference, displayed in Fig. 2 (a). 
Figure 2 - BPSK Signal Under Interference Effect

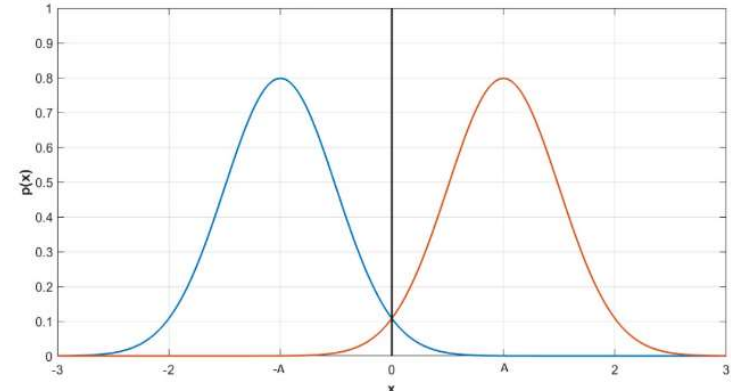

(a) Normal interference

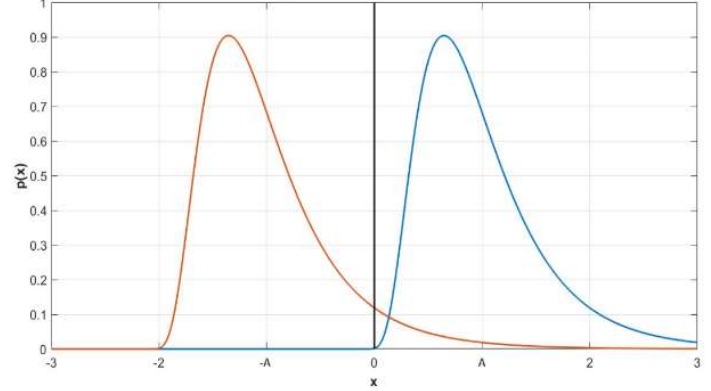

(b) Lognormal interference

Source: Prepared by the author

That distribution can be used to compute the symbol or bit error probability for different modulation schemes. The Lognormal probability density function is a heavy tail distribution, that is, it decays slower than an exponential. Therefore, there is an expected increase in the error probability during the power adaptation process.

In order to compute the bit error rate for a BPSK signal, in the case of the zero mean Gaussian distribution, it suffices to compute the following integral,

$$
P_{E}=\int_{0}^{\infty} \frac{1}{\sigma \sqrt{2 \pi}} e^{-\frac{(x-A)^{2}}{2 \sigma^{2}}} d x,
$$

in which $A$ is the modulated signal amplitude. The preceding equation can be rewritten, using the transformation, $t=(x+A) / \sigma, d x=\sigma d t$., to obtain the error probability,

$$
P_{E}=\int_{\frac{A}{\sigma}}^{\infty} \frac{1}{\sqrt{2 \pi}} e^{-\frac{1}{2} t^{2}} d t
$$

That can be simplified, using the $Q(\cdot)$ function, to

$$
P_{E}=Q\left(\frac{A}{\sigma}\right) \text {. }
$$

This puts into evidence the distribution symmetry, and the fact that the errors are equiprobable.

For a Lognormal distribution, which is asymmetric, the same fact does not occur. In this case, the influence of the interference that affects symbol $A$ in the computation of the error probability is higher. It approximates the total error probability in case that the transmission power is high.

For a low transmission power, the influence of the interference that occurs in symbol $-A$ becomes more evident, because the graphic of the Lognormal distribution is displaced to the left and can cross the coordinate axes. In this case, the error probability increases suddenly, causing a discontinuity in the curve.

The error probability for the transmission of symbol $-A$ is given by

$$
P_{E}(-A)=\frac{1}{2} \int_{0}^{\infty} \frac{1}{(x+A) \sigma \sqrt{2 \pi}} e^{-\left(\frac{\ln (x+A)}{2 \sigma}\right)^{2}} d x,
$$

in which $-A$ is the mean value of the distribution that results from the power probability density function transformation. 
This transformation, $X(t)=\sqrt{P(t)}$, displaces the interference to the origin of the coordinated system, and the transmitted signal has zero mean. Because of the asymmetry of the Lognormal distribution, the error probabilities are different for each symbol $P_{E}(-A) \neq P_{E}(A)$.

Monte Carlo simulation has been used to test the results, to compare with the analytical plots. The results are presented in Figs. 3 (a) to (d), for the BPSK modulation. It is possible to note, for simulated variance values, that the bit error probability obtained with the Lognormal distribution is always above that obtained from the Gaussian distribution, that represents the common noise. Also, the Lognormal BER curve decays at a slow rate, as compared to the Gauss BER plot.

Figure $3-B E R$ as a function of $A$ in BPSK modulation

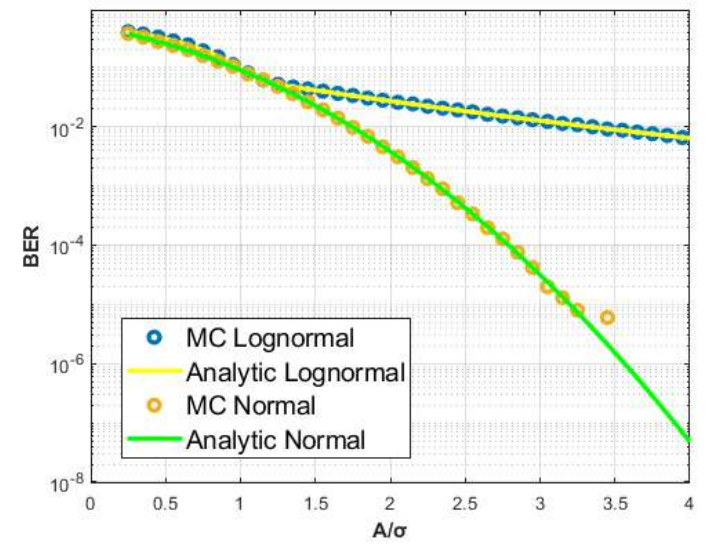

(a) With $\sigma=0,75$

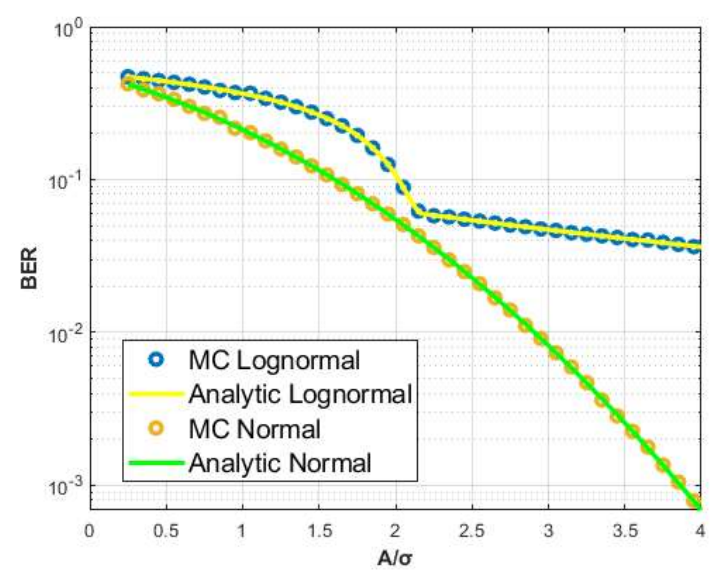

(c) With $\sigma=1,25$

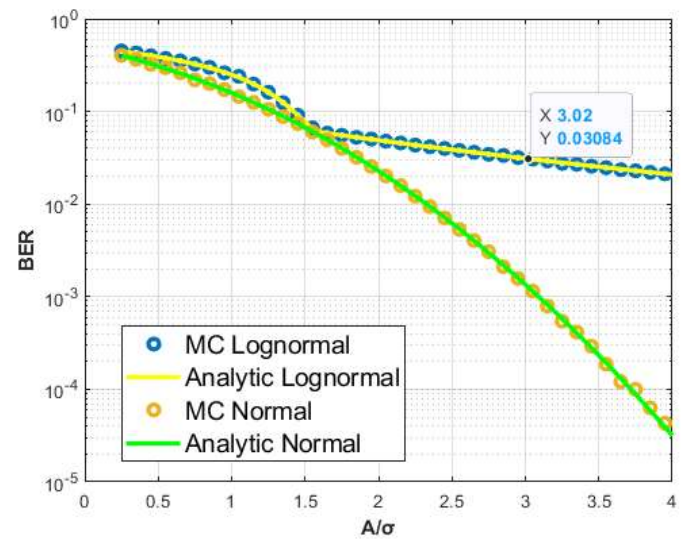

(b) With $\sigma=1,00$

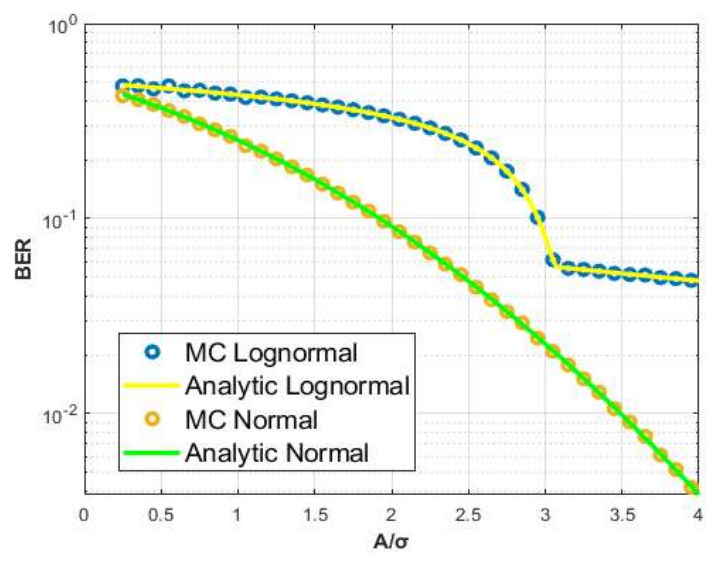

(d) With $\sigma=1,50$

Source: Prepared by the author

For such a non-stationary process, Itô integration has been used to solve the problem, which, for the defined constraints, resulted in a Lognormal probability density function, that, different from the Gaussian distribution, is asymmetrical. The long-term 
interference average and variance values follow exponential curves, which depend on certain system parameters.

\section{CONCLUSION}

It is usual to assume stationarity in the analysis of symbol or bit error probability, because the analysis of a non-stationary environment is mathematically complex.

But, in cellular communication systems, the interference is usually dependent on the state of the network, and if the users decide to access the network all of a sudden, the traffic modelling, as well as the interference, is non-stationary.

This article presented a mathematical modelling of the effect of an interference accumulation, caused by a sudden increase of users in a digital cellular system, also called an information outbreak or epidemic interference.

For such a heavy tail distribution, there is an expected increase in the error probability during the adaptation process.

\section{REFERENCES}

1. Sakagushi, Kei. et al. Millimeter-Wave Wireless LAN and Its Extension toward 5G Heterogeneous Networks. IEICE Trans. Commun., vol.E98-B, no.10, 2015.

2. Frederico, F. P. and Sabino, C. Á. V. 5 G New Radio Evolution, Challenges and Solutions. Rohde \& Schwarz, 2019.

3. Alencar, M. S., and Blake, I. F. 'The Capacity for a Discrete-State Code Division Multiple Access Channel'. IEEE Journal on Selected Areas in Communications 12, 5 (June 1994), 925- 937.

4. Brzezniak, Z., and Zastawniak, T. Basic Stochastic Processes. Springer, London, Great Britain, 2006.

5. Itô, K. 'Stochastic integral'. Proceedings of the Imperial Academy 8, 20 (1944), 519-524.

6. Itô, K. 'On a Stochastic Integral Equation'. Proceedings of the Imperial Academy 2, 22 (1946), 32-35.

7. Movellan, J. R. 'Tutorial on Stochastic Differential Equations'. Technical report, MPLab Tutorials Version 06.1, 2011. 